\title{
Ferning, Schimer I and Tear Break Up Time (Tbut) Accuracy Test in Post-Operative Cataract Patient with Extra Capsular Catarak Extraction (Ecce) Technique
}

\author{
Anang Tribowo', Lady Kavotiner ${ }^{1}$, Theodorus ${ }^{2}$, Alie Solahuddin ${ }^{1}$, Vidya Hestika ${ }^{3 *}$ \\ ${ }^{1}$ Departement of Ophthalmology, Faculty of Medicine, Moeh.Hoesin Hospital, Sriwijaya University, Indonesia. \\ ${ }^{2}$ Medical Research Unit, Faculty of Medicine, Sriwijaya University, Indonesia. \\ ${ }^{3}$ Fellow Students, Department of Ophthalmology, Faculty of Medicine, Sriwijaya University, Indonesia.
}

A R T I C L E I N F O
Keywords:
Accuration
Cataract
ECCE
Ferning Schirmer 1
TBUT
*Corresponding author:
Vidya Hestika
E-mail address:
disty.andryani@yahoo.com
All authors have reviewed and
approved the final version of the
manuscript.

https://doi.org/10.32539/bsm.v5i2.219

\begin{abstract}
A B S T R A C T
Objective: To determine the accuracy of the Ferning, Schimer I and TBUT test in cataract patients after cataract surgery with the ECCE technique at the Eye Polyclinic at Mohammad Hoesin Hospital Palembang and the Special Eye Hospital Palembang Methods: Diagnostic test was undertaken at the Eye Polyclinic at Mohammad Hoesin Hospital Palembang and the Special Eye Hospital Palembang from May 2019 to January 2020. There were 42 postoperative cataract patients who met the inclusion criteria. Ferning, Schimer I and TBUT tests were performed before surgery and one month after surgery. Data analysis using SPSS version 22.0 (SPSS, Inc Chicago, Ilinois). Results: Fourty two samples of postoperative cataract surgery with ECCE technique obtained by female sex $(57,1 \%)$ with a mean age of cataract patients of approximately 58.09 years. The accuracy of Schimer I and TBUT is 0.952 with $100 \%$ sensitivity, $50 \%$ specificity, $95 \%$ positive predictive value (PPV) and $100 \%$ negative predictive value (NPV), the accuracy of TBUT and Ferning is 0.881 with $90.2 \%$ sensitivity, $0 \%$ specificity , positive predictive value (PPV) $97.4 \%$ and negative predictive value (NPV) 0\% while the accuracy of Ferning and Schimer I was 0.928 with a sensitivity of $97.5 \%$, specificity of $0 \%$, positive predictive value (PPV) of $95.1 \%$ and negative predictive value (NPV) $0 \%$. Conclusion: Schimer I test is more accurate to use as a predictor of dry eye in postoperative cataract patients than Ferning and TBUT
\end{abstract}

\section{Introduction}

Dry Eye Syndrome is a multifactorial disorder of the surface of the eyeball and tears that causes discomfort, tear film instability, visual disturbances, potentially damaging the ocular surface. [1,2].

The prevalence of Dry Eye Syndrome increases with age, affecting about $5 \%$ of the adult population during the fourth decade of life, increasing to $10-15 \%$ in adults over 65 years of age. This is because in elderly patients there are pathological changes in the tear glands, which will cause disturbance in the dynamic of tears. In addition, at the age of over 60 years, there can be a decrease in the production of tears which will cause
Dry Eye Syndrome [1-4]

Examination in Dry Eye Syndrome is useful for assessing tear function in quality and quantity such as Ferning test, Schirmer test and Tear break-up time (TBUT). Ferning examination to assess the quality and stability of the tears. Schirmer I examination to assess the quantity of tear production produced by the lacrimal glands. Meanwhile, Tear Break Up Time is to assess the stability of the tear film. $[3,4]$

Cataract surgery is an operation involving the conjunctiva and cornea, aims to produce good vision but can cause symptoms of dry eye syndrome. One of 
the cataract surgery techniques is a corneosclera incision in Extra Capsular Cataract Extraction (ECCE).[5] Research by Zhang, et al. In 2010 reported that more than half of the samples who underwent cataract surgery using the ECCE method experienced postoperative Dry Eye Syndrome.[6]

This research will analyze accuracy of the Schirmer, TBUT, and Ferning examinations on the risk of dry eye syndrome in patients after cataract surgery using the Extra Capsular Cataract Extraction method. On this basis, this research was conducted with the hope of being selective in choosing cataract surgery procedures to be performed on cataract patients.

\section{Methods}

Diagnostic test was undertaken in May 2019 to January 2020 at the Eye Polyclinic at Mohammad Hoesin Hospital Palembang and the Special Eye Hospital Palembang. There were 42 postoperative cataract patients who met the inclusion and exclusion criteria. The protocol has been approved of Ethics Committee, Faculty of Medicine Sriwijaya University. Examination of Ferning, Schirmer 1 and TBUT test was done before and one month after cataract surgery. The frequency and distribution of postoperative cataract patient's data are described in univariate table and graph, and the accuracy of Ferning, Schirmer 1 and TBUT test were analysis using SPSS version 22.0 (SPSS, Inc Chicago, Mlinois).

\section{Results}

In the period May 2019 to January 2020 there were 42 postoperative cataract patients who meet the criteria of inclusion and exclusion.

Table 1 summarize that out of 42 postoperative cataract patients, there are 18 male (42.9\%) and 24 female $(57.1 \%)$ with average age $58.09 \pm 6.072$ years (age range 47-69 years). Patients with cataracts in this study the majority of higher education as much as $59.6 \%$ (senior high school and bachelor degree) and the majority of occupations in this study is retired (42.5\%).

The ophthalmological characteristics of the research subjects are shown in Table 2 and 3. In this study, majority of preoperative vision was $1 / 300$ (71.4\%) whereas after surgery the majority of vision was $6 / 9$ $(47.6 \%)$ and there were no more patients with $1 / 300$ vision. Before surgery, the Schimer I value was abnormal/positive for 3 patients (7.1\%) and increased one month after surgery to 40 people $(95.2 \%)$, the abnormal/positive TBUT value also increased after surgery from only 9 patients $(21.4 \%)$ to 38 patients $(90.5 \%)$ as well as abnormal/positive Ferning scores increased after surgery from initially there were no patients with abnormal/positive values to 41 patients $(97.6 \%)$.

Graphs 1,2 and 3 show a decrease in the value of Schirmer I and TBUT 1 one month after surgery.

Table 4 shows the comparison of the accuracy value of Ferning, Schimer I and TBUT to diagnose dry eye syndrom in postoperative cataract patients with ECCE technique. In this study, the accuracy of Schimer I and TBUT is 0.952 with $100 \%$ sensitivity, 50\% specificity, 95\% positive predictive value (PPV) and 100\% negative predictive value (NPV).

The accuracy of TBUT and Ferning was 0.881 with 90.2\% sensitivity, 0\% specificity, 97.4\% positive predictive value (PPV) and $0 \%$ negative predictive value (NPV). In addition, the accuracy of Ferning and Schimer I is 0.928 with a sensitivity of $97.5 \%$, a specificity of $0 \%$, a positive predictive value (PPV) of $95.1 \%$ and a negative predictive value (NPV) of $0 \%$.

The Youden Index Ferning Test value is -0.025 , the Youden Index Schimer I Test value is 0.500 and the Youden Index Test TBUT value is -0.080 . So it can be concluded that Schimer I is more accurate to be used as a predictor of dry eye in postoperative cataract patients than Ferning and TBUT. 
Table 1. Characteristics demographics of research subject

\begin{tabular}{llc}
\hline Characteristics & & Total \\
\hline Age (years) & Mean \pm SD & $58.09 \pm 6.072$ \\
& & $(47-69)$ \\
\hline Sex, n (\%) & Male & $18(42.9)$ \\
& Female & $24(57.1)$ \\
\hline Education, n(\%) & Elementary school & $6(14.3)$ \\
& Junior high school & $11(26.2)$ \\
& Senior high school & $12(28.6)$ \\
& Bachelor degree & $13(31.0)$ \\
\hline Occupation, $\mathrm{n}(\%)$ & Housewives & $7(16.7)$ \\
& Retired & $9(21.4)$ \\
& Laborers & $8(19.0)$ \\
& Farmers & $8(19.0)$ \\
& Traders & $4(9.5)$ \\
& Government employees & $6(14.3)$ \\
\hline
\end{tabular}

Table 2. Opthalmological characteristics of research subject before surgery

\begin{tabular}{lc}
\hline \multicolumn{1}{c}{ Characteristics } & Total \\
\hline Vision before surgery & $12(28.6)$ \\
$\bullet 1 / 60$ & $30(71.4)$ \\
$\bullet 1 / 30$ & $0(0.0)$ \\
\hline Ferning before surgery & $42(100.0)$ \\
$\bullet$ Abnormal & $3(7.1)$ \\
$\bullet$ Normal & $39(92.9)$ \\
\hline Schirmer I before surgery & \\
$\bullet$ Abnormal & $9(21.4)$ \\
\hline TBUT before surgery & $33(78.6)$ \\
\hline
\end{tabular}


Table 3. Opthalmological characteristics of research subject after surgery

\begin{tabular}{|c|c|}
\hline Characteristics & Total \\
\hline \multicolumn{2}{|c|}{ Vision after surgery } \\
\hline - $6 / 6$ & $1(2.4)$ \\
\hline - $6 / 7.5$ & $14(33.3)$ \\
\hline - $6 / 9$ & $20(47.6)$ \\
\hline - $6 / 12$ & $2(4.8)$ \\
\hline - $6 / 15$ & $2(4.8)$ \\
\hline - $1 / 60$ & $3(7.1)$ \\
\hline \multicolumn{2}{|c|}{ Ferning before surgery } \\
\hline - Abnormal & $41(97.6)$ \\
\hline - Normal & $1(2.4)$ \\
\hline \multicolumn{2}{|c|}{ Schirmer I after surgery } \\
\hline - Abnormal & $40(95.2)$ \\
\hline - Normal & $2(4.8)$ \\
\hline \multicolumn{2}{|c|}{ TBUT after surgery } \\
\hline - Abnormal & $38(97.6)$ \\
\hline - Normal & $4(2.4)$ \\
\hline
\end{tabular}

Tabel 4. Comparison of ferning, schimer I and TBUT accuracy value to diagnose dry eye syndrom in postoperative cataract patients with ecce technique

\begin{tabular}{lccc}
\hline & Ferning & Schimer I & TBUT \\
\hline Sensitivity, \% & 97.5 & 100 & 90.2 \\
Spesifisitas, \% & 0 & 50 & 0 \\
PPVe, \% & 0.951 & 0.950 & 0.974 \\
NPV, \% & 0 & 1 & 0 \\
Accuracy, \% & 0.928 & 0.952 & 0.881 \\
\hline
\end{tabular}




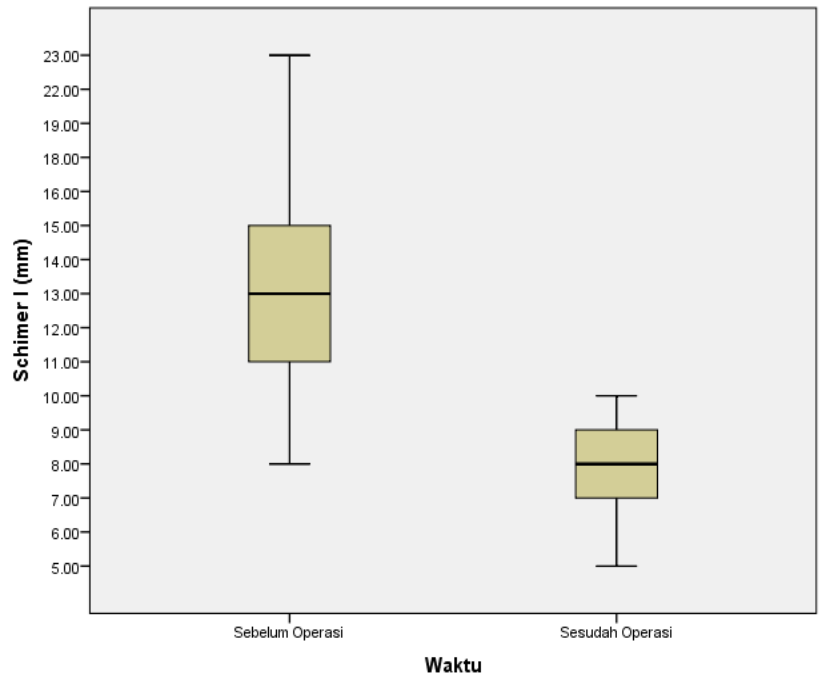

Graph 1. Schirmer I before and after surgery

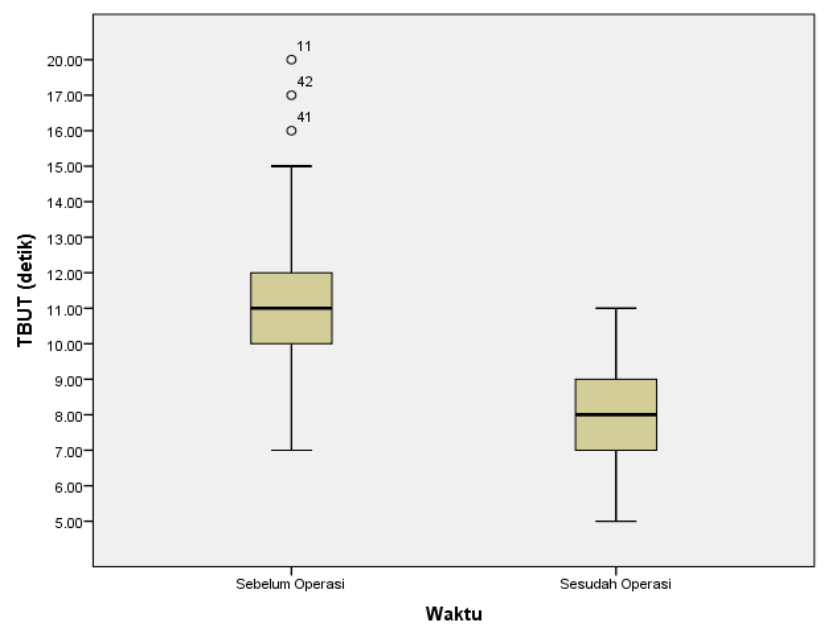

Graph 2. TBUT before and after surgery

\section{Discussion}

The mean age of cataract patients is approximately 58 years with an age range of 47-79 years and the majority of patients aged range 51 - 60 years $(47.6 \%)$. The results of this study are slightly. This result is slightly smaller than some previous studies. Chang et al in China (2013) involving 120 patients consisting of 60 cataract patients and 60 healthy controls reported that the mean age of cataract patients was $61 \pm 10.3$ years.[7] Likewise, the study of Usha et al in India (2014), out of 100 Cataract patients obtained a mean age of $60.92 \pm 9.5$ years. [8] The results of a study by Goyal et al in India in 2010 which involved 56 cataract patients showed that the mean age of senile cataract patients was $66.6 \pm 8.3$ years male sex and $62.4 \pm 10.0$ years in patients with female gender. [9]

In this study, the majority of cataract patients were female $(57.1 \%)$. This is supported by research conducted by Aminatul (2016) in Indonesia, of the 54 cataract patients who decided to have surgery, there were more female patients than men, 32 female patients $(53.3 \%)$, while the patient male number 28 (46.7\%).[10] Research Chang et al in China (2013) reported that out of 60 cataract patients, there were more female patients $(53.3 \%)$ than male patients (46.7\%).[7] A greater percentage of female sex was found in the study of Usha et al. In India (2014), from 100 cataract patients, $64 \%$ of patients were reported to 
be female (64 of 100 cataract patients).[8]

In this study, it was found that the vision of cataract patients was improved after surgery, before surgery the majority of patients had a vision of $1 / 300$ (71.4\%), but after surgery there was an increase in vision where the most visus was $6 / 9$ (47.6\%). It can be concluded that there is improvement in visual acuity after surgery compared to before surgery. The study of Ilechiel et al. (2012) in Ghana reported that there was an improvement in visual acuity after cataract surgery, before surgery, the most cataract patients' visus was $<3 / 60$ (99.7\%) but after surgery the highest visus was $6 / 24$ to $6 / 60(48,5 \%) \cdot[11]$

The ferning test is a test to assess the quality and stability of the tears. Crystallization patterns (ferning) are classified into 4 classes, types 1 and 2 are declared normal and types 3 and 4 are declared abnormal [12]. In this study, before surgery all cataract patients had normal ferning test scores (100\%) but one month after surgery $41 / 42$ patients $(97.6 \%)$ had abnormal ferning tests.

The Schirmer I examination is an assessment of the reflex secretion function of the lacrimal glands and the basal secretions from the Krause and Wolfring glands. A wetting $<10 \mathrm{~mm}$ after 5 minutes is diagnostic for aqueous layer deficiency [12]. In this study, the mean value of Schirmer I cataract patients before surgery was approximately $13.88 \mathrm{~mm}$ with the number of cataract patients with abnormal Schirmer I values only 3/42 patients $(7.1 \%)$ but one month after surgery $40 / 42$ patients (95.2\%) had abnormal Schirmer I tests.

The TBUT examination aims to assess the quality of the tears, in normal circumstances the tear film has a time of more than 10 seconds, whereas if it is less than 10 seconds it shows the tear layer is unstable [12]. In this study, the mean TBUT value of cataract patients before surgery was approximately 11.3 seconds, which means that the average patient had a stable tear film. The number of cataract patients with abnormal TBUT values was 9/42 patients (21.4\%). However, after 1 month of surgery, the number of cataract patients with abnormal TBUT values increased to $38 / 42$ patients (90.5\%).

With a diagnostic test based on normal values,
Schirmer I had 100\% sensitivity and 50\% specificity, this means that the ability of the Schirmer I value is not normal to detect abnormal TBUT values is only $100 \%$ while the ability of the normal Schirmer I value to detect $50 \%$ normal TBUT values. Patients with abnormal Schirmer I scores have a 95\% chance of having abnormal TBUT values and patients with normal Schirmer I scores have a 100\% chance of having normal TBUT values. The accuracy value of Schirmer I and TBUT is 0.952 which means that the degree of conformity of measurement (reliability) is very good.

Furthermore, with a diagnostic test based on normal values, the results obtained TBUT have a sensitivity of $90.2 \%$ and a specificity of $0 \%$, this means that the ability of the TBUT value is not normal to detect abnormal Ferning test values of $90.2 \%$ while the ability of normal TBUT values to detect values Normal Ferning test of $0 \%$. Normal TBUT values do not have the ability to predict normal Ferning test values, but as many as $97.4 \%$ of patients with abnormal TBUT will have abnormal Ferning test values. The accuracy value of TBUT and Ferning test is 0.881 which means that the degree of suitability of measurement (reliability) is very good.

In addition, with a diagnostic test based on normal values, it is found that Ferning has a sensitivity of $97.5 \%$ and a specificity of $0 \%$, this means that the ability of the Ferning value is not normal to detect an abnormal Schirmer I value of $95.2 \%$ while the ability of a normal Ferning value to detect a value Schirmer I is normal at $0 \%$. Normal Ferning values do not have the ability to predict normal Schirmer I values, but patients with abnormal Ferning values have a $97.5 \%$ chance of having abnormal Schirmer I values. The accuracy value of the Ferning and Schirmer I test is 0.928, which means that the degree of suitability of the measurement (reliability) is very good.

The Youden Index value is obtained by adding up the sensitivity and specificity values then subtracting 1 so that the Youden Index Ferning value is -0.025 , the Youden Index Schimer I value is 0.500 and the Youden Index TBUT is -0.080 . 


\section{Conclusion}

From these three examinations, it was found that all examinations had very good accuracy with the best accuracy obtained in the Schirmer I and TBUT tests. Meanwhile, based on the Youden index, Schirmer I had the greatest value. So it can be concluded that Schimer I is more accurate to be used as a predictor of dry eye in postoperative cataract patients than Ferning and TBUT.

\section{Acknowledgment}

We would like to thank Dr. Debby Handayati, M.Kes, for assistance the publication of this paper.

\section{References}

1. American Academy of Opthalmology. 2015. Basic and Clinical Science Course:120.Section 8-External Disease and Cornea, American Academy of Ophthalmology.

2. Phadatare, S.P et al. 2015. A Comprehensive Review on Dry Eye Disease: Diagnosis, Medical Management,Recent Development, and Future Challenges. (Online) (http://dx.doi.org/ $\underline{10.1155 / 2015 / 704946}$, diakses pada 1 Agustus 2017).

3. Stamper, L Robert, Marc F Lieberman, Michael V Drake. Becker-Shaffer's. Diagnosis and Theraphy of The Dry Eyes. Chapter 1. Mosby. Elsevier. 2009
4. Messmer, E.M. 2015. The Pathophysiology, Diagnosis, and Treatment of Dry Eye Disease. Deutsches Arzteblatt International 112(5): 71-81.

5. Retnaniadi S, Herwindo Dicky P. Effect of Type of Incision in Cataract Surgery on the occurrence of Dry Eye Syndrome. 2012. Medical Journal of Brawijaya. Vol. 27

6. Zhang S and Li YZ. Research of Ocular Surface Changes after Incisions of Cataract Surgery. International Journal of Ophthalmology (Guoji Yanke Zazhi). 2010; 10(9): 1719-1721
7. Chang D., Zhang X., Rong S., Sha Q., Liu P., Han T., dan Pan H. 2013. Serum Oxidative Enzyme Level and Oxidative Stress Product in Age-Related Cataract Patients. Oxidative Medicine and Cellular Longevity, 1-7. 2013

8. Usha S, Adline haris, et al. Serum Electrolytes in senile Cataract Patients. Al Ameen J Med Sci. 7(2):164-168. 2014

9. Goyal M.M., Vishwajeet P., Mittal R., dan Sune P. A Potential Correlation between Systemic Oxidative Stress and Intracellular Ambiance of the Lens Epithelia in Patients with Cataract. Journal of Clinical and Diagnostic Research, 4: 2061-2067. 2010.)

10. Aminatul Fitria. Relationship between Age, Attitude, Knowledge, Cost of Actions for Cataract Surgery. Periodic Journal of Epidemiology, Vol. 4 No. 2, May 2016: 176-187

11. Ilechie1 A.A, Kusi1 B,B, Ndudiri OV, Ofori1 EA. Evaluation of Post-Operative Visual Outcomes of Cataract Surgery in Ghana. International Journal of Health Research 2012 March; 5(1): 35-42

12. Blomquist, Preston H, Practical Ophthalmology: A Manual for Beginnng Residents, $7^{\text {th }}$ edition, American Academy of Ophthalmology: 2015. 